\title{
Observation of "Seesaw Effect" with Vancomycin, Teicoplanin, Daptomycin and Ceftaroline in 150 Unique MRSA Strains
}

Katie E. Barber • Cortney E. Ireland •

Natalia Bukavyn • Michael J. Rybak

To view enhanced content go to www.infectiousdiseases-open.com Received: November 22, 2013 / Published online: January 18, 2014

(C) The Author(s) 2014. This article is published with open access at Springerlink.com

\section{ABSTRACT}

Introduction: Vancomycin (VAN) failures associated with the treatment of complicated methicillin-resistant Staphylococcus aureus (MRSA) infections have been well described. The reported "seesaw effect" demonstrates improved $\beta$-lactam activity when VAN and/or daptomycin (DAP) susceptibility decreases. However, there are minimal data comparing ceftaroline (CPT) susceptibility with these agents or teicoplanin (TEI). Therefore, to further explore the seesaw effect, we evaluated the relationship between CPT and VAN, TEI, and DAP susceptibilities.

Methods: One hundred and fifty clinical MRSA isolates from the Anti-Infective Research Laboratory (Detroit, MI, USA) from 2008 to 2012 were analyzed. VAN, TEI, DAP and CPT

Electronic supplementary material The online version of this article (doi:10.1007/s40121-014-0023-0) contains supplementary material, which is available to authorized users.

K. E. Barber · C. E. Ireland · N. Bukavyn ·

M. J. Rybak $(\bowtie)$

Anti-Infective Research Laboratory, Pharmacy Practice-4148, Eugene Applebaum College of Pharmacy and Health Sciences, Wayne State University, 259 Mack Ave., Detroit, MI 48201, USA e-mail:m.rybak@wayne.edu minimum inhibitory concentrations (MIC) were determined via Etest methodology. $\mathrm{MIC}_{50}$ and $\mathrm{MIC}_{90}$ were calculated for each antibiotic. Additionally, four isogenic strain pairs were randomly selected for evaluation by time-kill methodology for the potential of enhanced killing by CPT as MICs increased to VAN, TEI, and DAP.

Results: CPT MICs were inversely correlated with VAN, DAP, and TEI MICs with correlation coefficients of $-0.535,-0.483$, and -0.386 , respectively $(P \leq 0.05)$. Comparison of the MIC relationship for glycopeptides and lipopeptides resulted in a positive correlation for all agent combinations. In time-kill evaluations, CPT demonstrated greater reductions in $\log _{10}$ colony-forming unit (CFU)/mL against mutant strains $\quad(3.73 \pm 0.67) \quad$ versus parents $(2.79 \pm 0.75)$ despite no change in CPT MIC $(P=0.112)$.

Conclusion: This study demonstrated a marked "seesaw effect" whereby CPT displayed increased susceptibility as the VAN, DAP, and TEI MICs increased. Additionally, we observed a positive linear correlation between VAN, DAP, and TEI MICs for all agent combinations. Enhanced activity was noted with CPT in 
mutant strains versus the parent strains despite no change in MIC. Based upon the enhanced CPT activity observed against strains with decreased susceptibility to VAN, DAP and TEI, CPT may provide an option for infections with reduced susceptibility to glycopeptides or lipopeptides. Further evaluation is warranted to investigate the clinical implications of the seesaw effect.

Keywords: Ceftaroline; Daptomycin; hVISA; Infection; MRSA; Seesaw effect; Staphylococcus aureus; Teicoplanin; Vancomycin; VISA

\section{INTRODUCTION}

Staphylococcus aureus continues to be a major healthcare threat. Methicillin-resistant $S$. aureus (MRSA) demonstrating reduced susceptibility to glycopeptides and lipopeptides such as vancomycin (VAN), teicoplanin (TEI), and daptomycin (DAP) severely limits our therapeutic options for treating complicated infections due to this pathogen. MRSA now comprises $55.5 \%$ of hospital-acquired $S$. aureus infections $[1,2]$. MRSA with reduced susceptibility to glyco- and lipopeptide antibiotics is increasingly being reported. Infections caused by MRSA isolates with reduced VAN susceptibility often lead to worse clinical outcomes, especially in strains identified as VAN-intermediate $S$. aureus (VISA), heterogeneous VISA (hVISA), or DAP non-susceptible (DNS) [3-10]. However, relatively few new antimicrobial agents are available, necessitating alternative treatment strategies including combination therapies and dose optimization as well as maximization of older antimicrobials. One newer strategy is based on the "seesaw effect", whereby $\beta$-lactam susceptibility increases as glyco- and lipopeptide susceptibility decreases, allowing for older beta-lactam antimicrobials to be used in combination with glycopeptides or lipopeptide antibiotics such as VAN and DAP or the anti-MRSA cephalosporin ceftaroline (CPT) $[11,12]$. However, this phenomenon has only been evaluated on a limited number of strains [12-16]. Therefore, the objective of this study was to further explore the "seesaw effect" in 150 clinical strains with varying susceptibilities. Additionally, eight strains were utilized in time-kill studies to determine if the response to CPT was affected by changing glycoor lipopeptide susceptibilities in isogenic strain pairs.

\section{MATERIALS AND METHODS}

\section{Bacterial Strains}

A total of 150 clinical MRSA strains from the Anti-infective Research Laboratory (Detroit, MI, USA) collected between 2008 to 2012 were chosen for evaluation of the "seesaw effect". All strains were randomly chosen clinical blood isolates. Additionally, four isogenic strain pairs were selected for further evaluation of these antibiotics in time-kill curves to compare differences in kill between parent and reduced susceptibility to VAN mutant isolates.

\section{Antimicrobials}

Ceftaroline $\left(\right.$ Teflaro $^{\circledR}$ ) powder was provided by Forest Laboratories, Inc. (New York, NY, USA). DAP (Cubicin ${ }^{\circledR}$ ) was purchased commercially from Cubist Pharmaceuticals (Lexington, MA, USA). VAN and TEI were purchased commercially from Sigma Chemical Co. (St. Louis, MO, USA). 


\section{Media}

Due to the calcium-dependent mechanism of DAP, MHB was supplemented with $50 \mathrm{mg} / \mathrm{L}$ of calcium and $12.5 \mathrm{mg} / \mathrm{L}$ of magnesium for all experiments. Colony counts were determined using tryptic soy agar (TSA) (Difco, Detroit, MI, USA).

\section{Susceptibility Testing}

Minimum inhibitory concentrations (MIC) for all study antimicrobials were determined by Etest methods according to the manufacturer's instructions. Additionally, broth microdilution MICs were performed in duplicate at $1 \times 10^{6}$ according to Clinical and Laboratory Standards Institute (CLSI) guidelines for isogenic strain pairs as a comparison/validation of MICs determined by Etest methodology [18]. All samples were incubated at $37^{\circ} \mathrm{C}$ for $18-24 \mathrm{~h}$. The following MIC data were determined for each tested antimicrobial: average $\mathrm{MIC}, \mathrm{MIC}_{50}$, and $\mathrm{MIC}_{90}$. These MIC data were analyzed by linear regression to derive correlations coefficients between agents.

\section{In Vitro Time-Kills}

Four isogenic strain pairs were chosen as representative strains for evaluation in timekill curves. Briefly, macro-dilution time-kill experiments were performed in duplicate using a starting inoculum of approximately $1 \times 10^{6} \mathrm{CFU} / \mathrm{mL}$ as previously described [1719]. The 24-well culture plate was utilized with $100 \mu \mathrm{L}$ of antibiotic stock solution, $200 \mu \mathrm{L}$ of a 1:10 dilution of a 0.5 McFarland standard organism suspension, and sufficient volume of CAMHB for a total volume of $2 \mathrm{~mL}$. Sample aliquots $(0.1 \mathrm{~mL})$ were removed over $0-24 \mathrm{~h}$ and serially diluted in cold $0.9 \%$ sodium chloride. Bacterial counts were determined using an automatic spiral plater (WASP; DW Scientific, West Yorkshire, UK) and colonies were enumerated using the protocol colony counter (Synoptics Limited, Frederick, MD, USA). If the anticipated dilution was near the MIC, vacuum filtration was used to avoid antibiotic carryover. Filtered samples were washed through a $0.45-\mu \mathrm{m}$ filter with normal saline to remove the antimicrobial agent. For both methods, plates were incubated at $37^{\circ} \mathrm{C}$ for $18-24 \mathrm{~h}$ at which time colony counts were performed. These methods have a lower limit of reliable detection of $1 \log _{10} \mathrm{CFU} / \mathrm{mL}$. Each isolate (parent and mutant) was tested against CPT, DAP, VAN, and TEI at the following human-simulated pharmacokinetic concentrations: free DAP peak $4.6 \mathrm{mg} / \mathrm{L}$ (equivalent to $4 \mathrm{mg} / \mathrm{kg} / \mathrm{day}, \quad 92 \%$ protein binding), free CPT midpoint concentration $3.5 \mathrm{mg} / \mathrm{L}$ (equivalent to $600 \mathrm{mg}$ every $12 \mathrm{~h}$; $20 \%$ protein binding), free VAN $7.5 \mathrm{mg} / \mathrm{L}$ (equivalent to $15 \mathrm{mg} / \mathrm{L}$ trough; $50 \%$ protein binding), and TEI trough $2 \mathrm{mg} / \mathrm{L}$ (equivalent to $20 \mathrm{mg} / \mathrm{L}$ trough; $90 \%$ protein binding). Timekill curves were graphed plotting the mean colony counts $\left(\log _{10} \mathrm{CFU} / \mathrm{mL}\right)$ versus time. Bactericidal activity was defined as $\geq 3 \log _{10}$ $\mathrm{CFU} / \mathrm{mL}$ (99.9\%) reduction from the starting inoculum. Bacteriostatic activity is defined as a 0 to $<3-\log _{10} \mathrm{CFU} / \mathrm{mL}$ reduction in colony count from the initial inoculum.

\section{Statistical Analysis}

Differences in $\log _{10} \mathrm{CFU} / \mathrm{mL}$ were analyzed by analysis of variance with Tukey's post hoc test. Correlation coefficients were determined via Spearman's rho testing. $P<0.05$ was considered significant. All statistical analyses were performed using SPSS statistical software (release 21.0; SPSS, Inc., Chicago, IL, USA). 


\section{Compliance with Ethics}

This article does not contain any studies with human or animal subjects performed by any of the authors.

\section{RESULTS}

A summary of MIC data is listed in Table 1. There was a large range of susceptibilities noted for each antimicrobial with DAP, TEI, and VAN having the largest range of susceptibilities. Positive MIC correlations were found between all glyco- and lipopeptides, VAN, DAP, and TEI. Inverse MIC correlations were found between CPT and all other agents. The correlation coefficients are listed in Table 2. MICs for the isogenic strains are listed in Table 3. In three of four pairs (D592 and D712, R6911 and R6913, A8090 and A8091), CPT activity was significantly more active against MRSA strains with reduced glycopeptide susceptibility despite the mutant strains having the same CPT MIC as the parent strains $(P=0.007,0.001,0.045)$. Against the 4th strain pair (R6491 and R6387), CPT demonstrated slightly improved activity against the mutant strain with a $4.3 \pm 0.3 \log _{10} \mathrm{CFU} / \mathrm{mL}$ reduction versus $3.76 \pm 0.3 \log _{10} \mathrm{CFU} / \mathrm{mL}$ reduction observed

Table 1 Minimum inhibitory concentration (MIC) (Etest) data summary

\begin{tabular}{llll}
\hline & $\begin{array}{l}\text { MIC range } \\
(\mathbf{m g} / \mathbf{L})\end{array}$ & $\begin{array}{l}\mathbf{M I C}_{50} \\
(\mathbf{m g} / \mathbf{L})\end{array}$ & $\begin{array}{l}\mathbf{M I C}_{\mathbf{9 0}} \\
(\mathbf{m g} / \mathbf{L})\end{array}$ \\
\hline CPT & $0.125-1.5$ & 0.38 & 1 \\
DAP & $0.03-4$ & 0.25 & 2 \\
TEI & $0.25-16$ & 1.5 & 8 \\
VAN & $0.19-8$ & 1 & 6 \\
\hline
\end{tabular}

$C P T$ ceftaroline, DAP daptomycin, TEI teicoplanin, VAN vancomycin
Table 2 Correlation coefficients

\begin{tabular}{|c|c|c|c|}
\hline & $\begin{array}{l}R \text { compared } \\
\text { to VAN }\end{array}$ & $\begin{array}{l}R \text { compared } \\
\text { to TEI }\end{array}$ & $\begin{array}{l}\mathrm{R} \text { compared } \\
\text { to DAP }\end{array}$ \\
\hline \multicolumn{4}{|l|}{ CPT } \\
\hline $\mathrm{MIC}_{90}$ & $-0.912^{*}$ & $-0.963^{*}$ & $-0.936^{*}$ \\
\hline $\mathrm{MIC}_{50}$ & $-0.858^{*}$ & $-0.847^{*}$ & $-0.818^{*}$ \\
\hline MIC & $-0.535^{*}$ & $-0.386^{*}$ & $-0.483^{*}$ \\
\hline \multicolumn{4}{|l|}{ DAP } \\
\hline $\mathrm{MIC}_{90}$ & $0.943^{*}$ & $0.947^{*}$ & - \\
\hline $\mathrm{MIC}_{50}$ & $0.959^{*}$ & $0.957^{*}$ & - \\
\hline MIC & $0.666^{*}$ & $0.632^{*}$ & - \\
\hline \multicolumn{4}{|l|}{ TEI } \\
\hline $\mathrm{MIC}_{90}$ & $0.971^{*}$ & - & - \\
\hline $\mathrm{MIC}_{50}$ & $0.997^{*}$ & - & - \\
\hline MIC & $0.789^{*}$ & - & - \\
\hline $\begin{array}{l}C P T \text { cef } \\
\text { inhibitory } \\
\text { vancomyc } \\
{ }^{*} P<0.05\end{array}$ & \multicolumn{3}{|c|}{$\begin{array}{l}\text { oline, } D A P \text { daptomycin, } M I C \text { minimum } \\
\text { concentration, } T E I \text { teicoplanin, } V A N\end{array}$} \\
\hline
\end{tabular}

Table 3 Minimum inhibitory concentrations for isogenic strain pairs

\begin{tabular}{lllll}
\hline Strain pairs & \multicolumn{4}{l}{ MICs $(\mathbf{m g} / \mathrm{L})$ parent/mutant } \\
\cline { 2 - 5 } & CPT & DAP & TEI & VAN \\
\hline R6911/R6913 & $0.5 / 0.5$ & $2 / 4$ & $4 / 4$ & $2 / 8$ \\
R6491/R6387 & $1 / 1$ & $0.5 / 0.5$ & $0.125 / 4$ & $1 / 2$ \\
D592/D712 & $1 / 1$ & $0.5 / 4$ & $0.5 / 2$ & $2 / 4$ \\
A8090/A8091 & $0.5 / 0.5$ & $0.25 / 1$ & $0.5 / 4$ & $1 / 8$ \\
\hline
\end{tabular}

$C P T$ ceftaroline, DAP daptomycin, TEI teicoplanin, $V A N$ vancomycin

for the parent, though this was not statistically significant $\quad(P=0.318)$ Overall, CPT demonstrated greater activity against all mutant strains with an average of $3.73 \pm 0.67 \log _{10} \mathrm{CFU} / \mathrm{mL}$ reduction in mutant strains versus $2.79 \pm 0.75 \log _{10} \mathrm{CFU} / \mathrm{mL}$ 
reduction for the parent strains with no change in CPT susceptibility noted $(P=0.112)$ as illustrated in Fig. 1. DAP demonstrated potent bactericidal activity against all susceptible strains with a $\log _{10} \mathrm{CFU} / \mathrm{mL}$ decrease of $3.5 \pm 0.8 \log _{10} \mathrm{CFU} / \mathrm{mL}$. A bactericidal effect was also noted for two mutant strains (D712 and A8091). However, after the initial kill within the first $8 \mathrm{~h}$, significant regrowth of 1.5 $\log _{10} \mathrm{CFU} / \mathrm{mL}$ increase from starting inoculum occurred in the other two mutants. VAN demonstrated activity against all parent isolates within the first $8 \mathrm{~h}$, but kill was not sustained over the complete duration of the experiment against R6491. Against R6387, VAN demonstrated bacteriostatic activity with

a R6911 and R6913
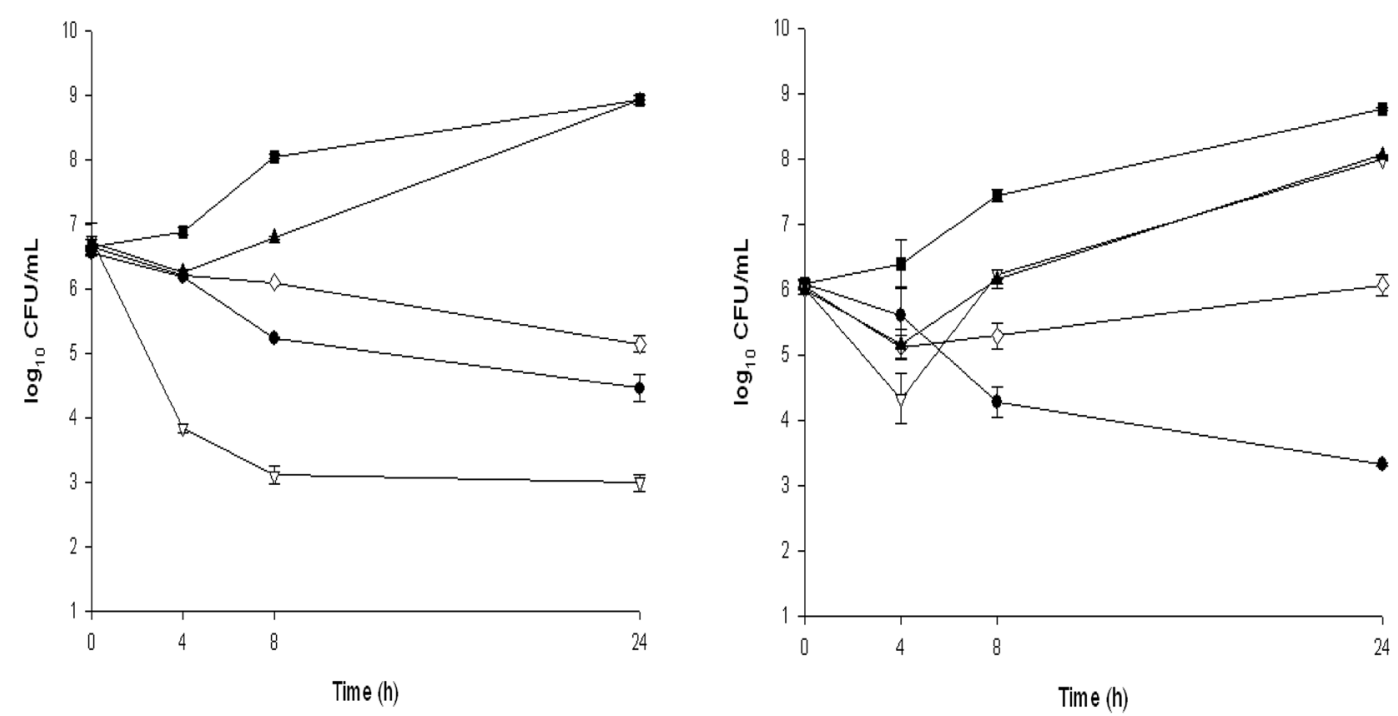

b R6491 and R6387
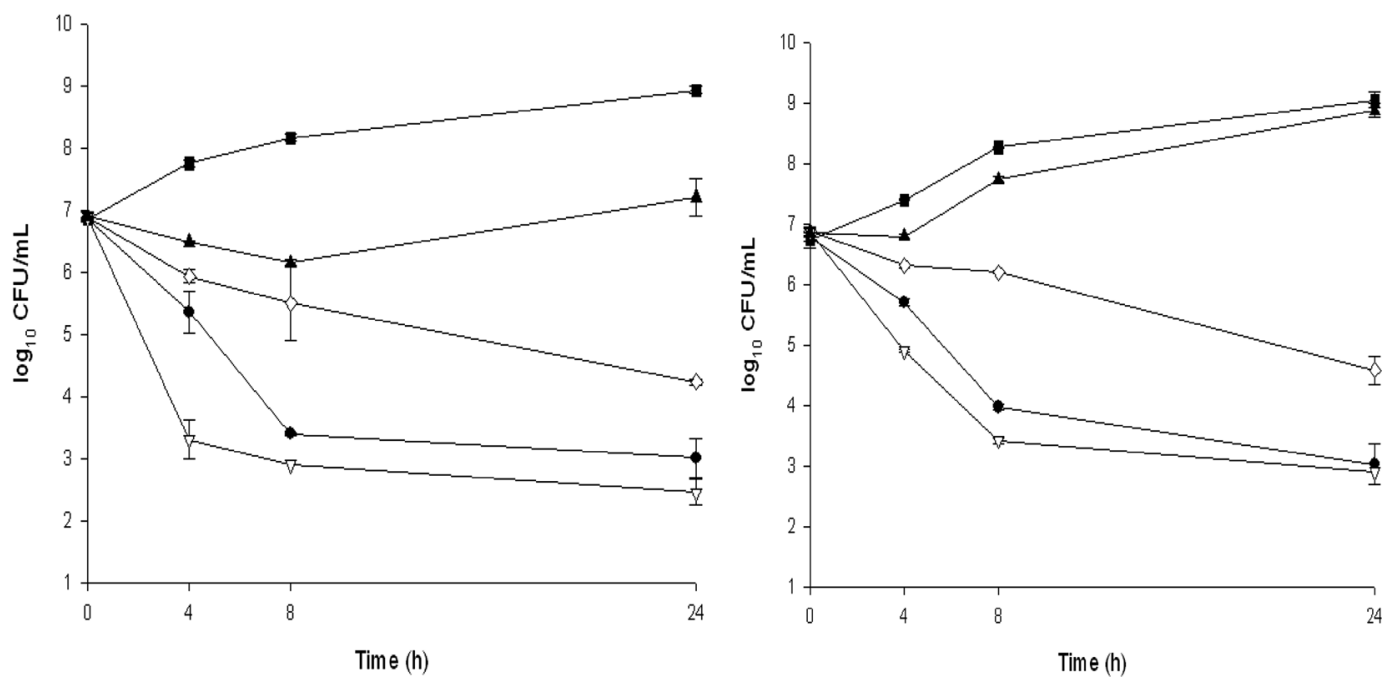

Fig. 1 Time-kill evaluation results. Closed circles ceftaroline, open triangles daptomycin, closed triangles teicoplanin, open diamonds vancomycin, closed squares drug-free control 

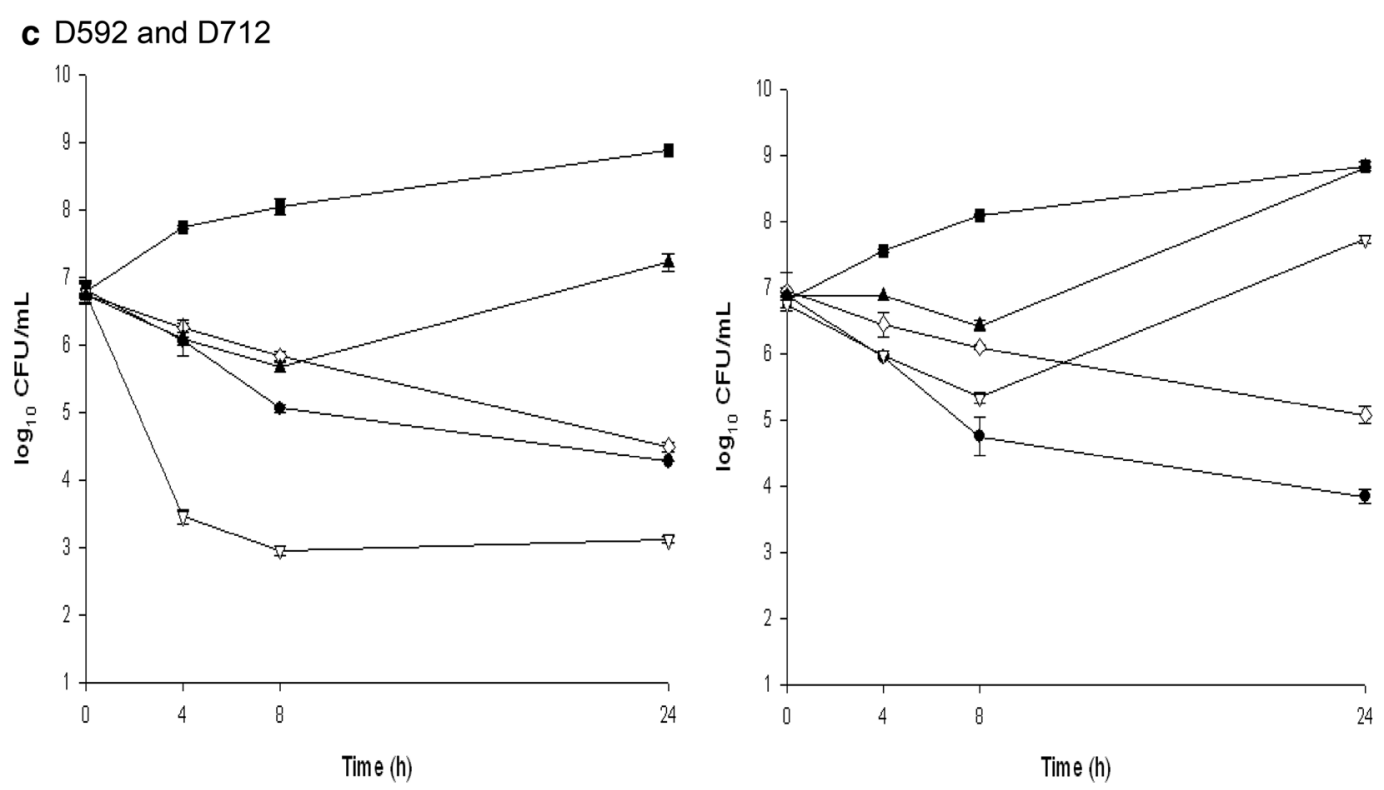

d $\mathrm{A} 8090$ and $\mathrm{A} 8091$
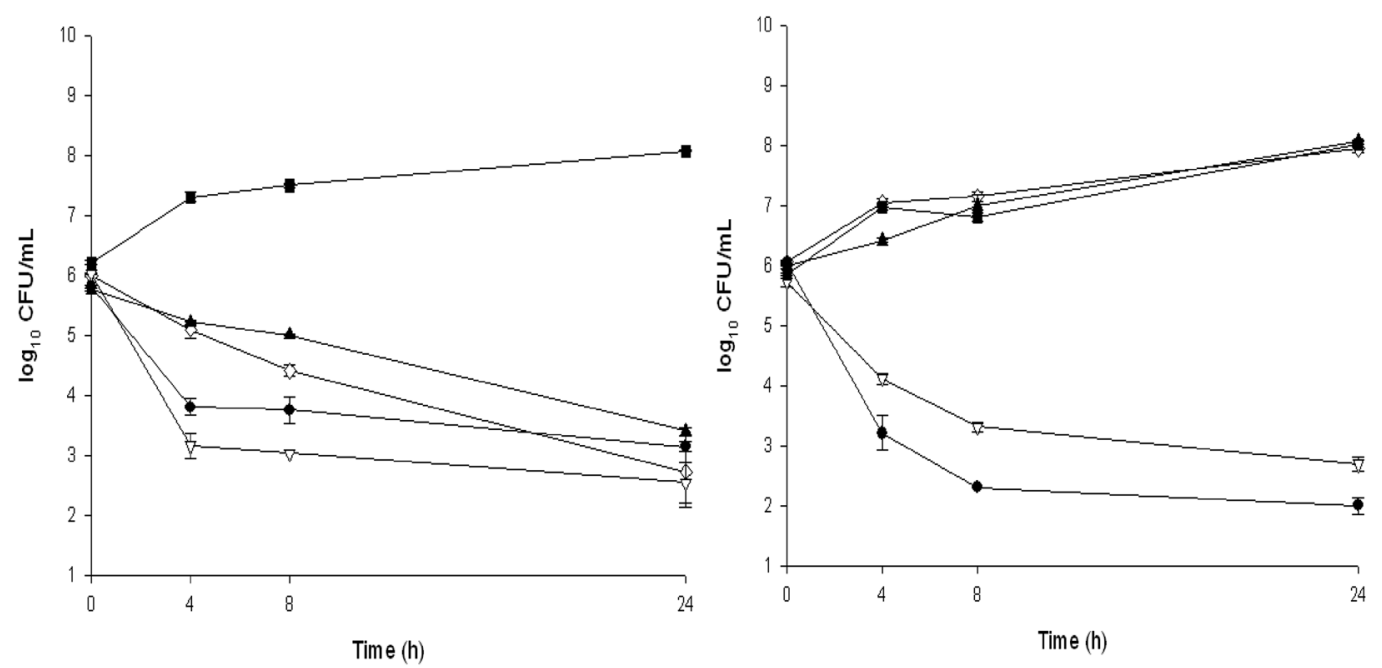

Fig. 1 continued

$2.3 \pm 0.1 \log _{10} \mathrm{CFU} / \mathrm{mL}$ reduction, but no appreciable activity was noted against any of the other mutants. TEI only displayed activity against one of the eight strains tested (A8090) with $2.4 \pm 0.1 \log _{10} \mathrm{CFU} / \mathrm{mL}$ reduction over $24 \mathrm{~h}$. All remaining strains with TEI demonstrated minimal to no activity $(0-<1$ $\log _{10} \mathrm{CFU} / \mathrm{mL}$ reduction).

\section{DISCUSSION}

The results of this study demonstrate that as the VAN MIC increased, a linear increase in MIC was also observed for DAP and TEI. This positive correlation was more pronounced with the two glycopeptides, but was only slightly less for DAP. Although not previously reported with 
TEI, we observed the same "seesaw effect" with TEI that has previously been demonstrated with VAN and DAP [15]. Additionally, the CPT MIC appeared to decrease as the glyco- and lipopeptide MIC increased. In our time-kill evaluations, СPT was more active against isolates with reduced susceptibility to glycoand lipopeptide antimicrobials than to the parent strains. Of note, the CPT MIC did remain the same from parent to mutant, while the MIC for the other agents increased. For the parent isolates, CPT demonstrated consistent kill compared to mutants, though DAP appeared to have the most pronounced activity for all parent isolates.

The "seesaw effect" was first reported as a laboratory phenomenon by Sieradzki and colleagues [16]. The parent isolate, COL, had a methicillin MIC of $800 \mathrm{mg} / \mathrm{L}$ with a VAN MIC of $1.5 \mathrm{mg} / \mathrm{L}$; after exposing the isolate to in vitro VAN pressure, MIC increased from 1.5 and $100 \mathrm{mg} / \mathrm{L}$, respectively. The first clinical case describing this type of effect was published 2 years later in a 79-year-old hemodialysis patient with MRSA bacteremia [13]. Initial isolates obtained demonstrated an oxacillin MIC of $3 \mathrm{mg} / \mathrm{L}$ and a VAN MIC of $2 \mathrm{mg} / \mathrm{L}$. After continued VAN exposure and documented sub-therapeutic VAN serum concentrations, the VAN MIC increased to $8 \mathrm{mg} / \mathrm{L}$ whereas the oxacillin MIC subsequently decreased to $0.8 \mathrm{mg} / \mathrm{L}$. Similarly, a second case report was published describing a similar effect in a patient with MRSA-infective endocarditis [14]. This patient received a prolonged course of VAN therapy, and as therapy continued the VAN MIC increased from 1 to $8 \mathrm{mg} / \mathrm{L}$ while the oxacillin MIC decreased from as high as 100 to $0.75 \mathrm{mg} / \mathrm{L}$.

Additional research on this phenomenon has been carried out utilizing pharmacokinetic/pharmacodynamics in vitro modeling. Werth and colleagues [15] performed in vitro studies evaluating three isogenic $S$. aureus strain pairs, including DNS and VISA strains exposed to human-simulated concentrations of CPT and VAN. In all three pairs, CPT activity was significantly more active against MRSA strains with reduced glycopeptide susceptibility despite the mutant strains having the same CPT MIC as the parent strains.

Though there are in vitro and in vivo data to support the "seesaw effect", this is the first study to evaluate such a large number of strains including a significant number that are unrelated (all strains except the 8 isogenic strains). The sample of 150 isolates demonstrated a seesaw pattern. These data help to confirm the previous observations that have been reported with a few clinical or laboratory-derived strains.

As resistance has emerged to antibiotics such as VAN and DAP, the seesaw effect may provide an avenue for alternative therapeutic options. The seesaw effect can also be further exploited through combination therapy of a glyco- or lipopeptide plus an anti-staphylococcal betalactam. In the presence of an antistaphylococcal $\beta$-lactam, DAP binding is increased leading to enhanced depolarization despite increases in DAP MIC [11, 20].

\section{Limitations}

Potential limitations for this investigation include the evaluation of a limited number of strains and antibiotic combinations utilized in the time-kill curve assessments. Additionally, time-kill curve methodology only utilizes fixed concentration exposures. To further elicit additional impact, multiple dose pharmacokinetic modeling would need to be analyzed. 


\section{CONCLUSION}

In 150 isolates, it was evident that CPT MICs decreased as VAN, TEI, and DAP MICs increased. After additional testing on eight isogenic strains, enhanced CPT killing was observed in the strains with decreased susceptibility to VAN, DAP, and TEI. CPT may provide clinicians with a therapeutic alternative due to enhanced activity when faced with MRSA isolates with elevated glyco- or lipopeptide MICs, such as hVISA, VISA, or DNS strains. However, additional research is warranted to determine the clinical utility of this phenomenon.

\section{ACKNOWLEDGMENTS}

No funding or sponsorship was received for this study or publication of this article.

MJR has received grant support, consulted for, or provided lectures for Cubist, Durata, Forest, Novartis and Sunovion, Theravance and funding in part by NIH NIAID R21A109205501. KEB, CEI, and NB have no potential conflicts of interest to declare.

We thank George Sakoulas for providing strains (A8090, A8091, D592, and D712) for this research.

Michael J. Rybak is the guarantor for this article and takes responsibility for the integrity of the work as a whole.

Compliance with ethics. This article does not contain any studies with human or animal subjects performed by any of the authors.

Open Access. This article is distributed under the terms of the Creative Commons Attribution Noncommercial License which permits any noncommercial use, distribution, and reproduction in any medium, provided the original author(s) and the source are credited.

\section{REFERENCES}

1. Sievert DM, Ricks P, Edwards JR, Schneider A, Patel J, Srinivasan A, et al. Antimicrobial-resistant pathogens associated with healthcare-associated infections: summary of data reported to the National Healthcare Safety Network at the Centers for Disease Control and Prevention, 2009-2010. Infect Control Hosp Epidemiol. 2013;34(1):1-14 (Epub 2012/12/12).

2. Hidron AI, Edwards JR, Patel J, Horan TC, Sievert DM, Pollock DA, et al. NHSN annual update: antimicrobial-resistant pathogens associated with healthcare-associated infections: annual summary of data reported to the National Healthcare Safety Network at the Centers for Disease Control and Prevention, 2006-2007. Infect Control Hosp Epidemiol. 2008;29(11):996-1011 (Epub 2008/10/ 25).

3. van Hal SJ, Paterson DL. Systematic review and meta-analysis of the significance of heterogeneous vancomycin-intermediate Staphylococcus aureus isolates. Antimicrob Agents Chemother. 2011;55(1):405-10 (Epub 2010/11/17).

4. Sakoulas G, Moise-Broder PA, Schentag J, Forrest A, Moellering RC Jr, Eliopoulos GM. Relationship of MIC and bactericidal activity to efficacy of vancomycin for treatment of methicillin-resistant Staphylococcus aureus bacteremia. J Clin Microbiol. 2004;42(6):2398-402 (Epub 2004/06/09).

5. Neoh HM, Hori S, Komatsu M, Oguri T, Takeuchi F, Cui L, et al. Impact of reduced vancomycin susceptibility on the therapeutic outcome of MRSA bloodstream infections. Ann Clin Microbiol Antimicrob. 2007;6:13 (Epub 2007/10/31).

6. Lodise TP, Graves J, Evans A, Graffunder E, Helmecke M, Lomaestro BM, et al. Relationship between vancomycin MIC and failure among patients with methicillin-resistant Staphylococcus aureus bacteremia treated with vancomycin. Antimicrob Agents Chemother. 2008;52(9): 3315-20 (Epub 2008/07/02).

7. Soriano A, Marco F, Martinez JA, Pisos E, Almela M, Dimova VP, et al. Influence of vancomycin minimum inhibitory concentration on the treatment of methicillin-resistant Staphylococcus aureus bacteremia. Clin Infect Dis. 2008;46(2):193-200 (Epub 2008/01/04). 
8. Musta AC, Riederer K, Shemes S, Chase P, Jose J, Johnson LB, et al. Vancomycin MIC plus heteroresistance and outcome of methicillinresistant Staphylococcus aureus bacteremia: trends over 11 years. J Clin Microbiol. 2009;47(6):1640-4 (Epub 2009/04/17).

9. Wang JL, Wang JT, Sheng WH, Chen YC, Chang SC. Nosocomial methicillin-resistant Staphylococcus aureus (MRSA) bacteremia in Taiwan: mortality analyses and the impact of vancomycin, $\mathrm{MIC}=2 \mathrm{mg} / \mathrm{L}$, by the broth microdilution method. BMC Infect Dis. 2010;10:159 (Epub 2010/06/10).

10. Kullar R, Davis SL, Levine DP, Rybak MJ. Impact of vancomycin exposure on outcomes in patients with methicillin-resistant Staphylococcus aureus bacteremia: support for consensus guidelines suggested targets. Clin Infect Dis. 2011;52(8): 975-81 (Epub 2011/04/05).

11. Dhand A, Bayer AS, Pogliano J, Yang SJ, Bolaris M, Nizet $V$, et al. Use of antistaphylococcal betalactams to increase daptomycin activity in eradicating persistent bacteremia due to methicillin-resistant Staphylococcus aureus: role of enhanced daptomycin binding. Clin Infect Dis. 2011;53(2):158-63 (Epub 2011/06/22).

12. Mwangi MM, Wu SW, Zhou Y, Sieradzki K, de Lencastre $\mathrm{H}$, Richardson $\mathrm{P}$, et al. Tracking the in vivo evolution of multidrug resistance in Staphylococcus aureus by whole-genome sequencing. Proc Natl Acad Sci USA. 2007;104(22): 9451-6 (Epub 2007/05/23).

13. Sieradzki K, Roberts RB, Haber SW, Tomasz A. The development of vancomycin resistance in a patient with methicillin-resistant Staphylococcus aureus infection. N Engl J Med. 1999;340(7):517-23 (Epub 1999/02/18).

14. Sieradzki K, Leski T, Dick J, Borio L, Tomasz A. Evolution of a vancomycin-intermediate Staphylococcus aureus strain in vivo: multiple changes in the antibiotic resistance phenotypes of a single lineage of methicillin-resistant $S$. aureus under the impact of antibiotics administered for chemotherapy. J Clin Microbiol. 2003;41(4): 1687-93 (Epub 2003/04/12).

15. Werth BJ, Steed ME, Kaatz GW, Rybak MJ. Evaluation of ceftaroline activity against heteroresistant vancomycin-intermediate Staphylococcus aureus and vancomycinintermediate methicillin-resistant $S$. aureus strains in an in vitro pharmacokinetic/pharmacodynamic model: exploring the "seesaw effect". Antimicrob Agents Chemother. 2013;57(6):2664-8 (Epub 2013/04/03).

16. Sieradzki K, Tomasz A. Inhibition of cell wall turnover and autolysis by vancomycin in a highly vancomycin-resistant mutant of Staphylococcus aureus. J Bacteriol. 1997;179(8):2557-66 (Epub 1997/04/01).

17. Werth BJ, Vidaillac C, Murray KP, Newton KL, Sakoulas G, Nonejuie P, et al. Novel combinations of vancomycin plus ceftaroline or oxacillin against methicillin-resistant vancomycin-intermediate Staphylococcus aureus (VISA) and heterogeneous VISA. Antimicrob Agents Chemother. 2013;57(5): 2376-9 (Epub 2013/02/21).

18. Vidaillac C, Parra-Ruiz J, Rybak MJ. In vitro timekill analysis of oritavancin against clinical isolates of methicillin-resistant Staphylococcus aureus with reduced susceptibility to daptomycin. Diagn Microbiol Infect Dis. 2011;71(4):470-3 (Epub 2011/10/25).

19. Leonard SN, Kaatz GW, Rucker LR, Rybak MJ. Synergy between gemifloxacin and trimethoprim/ sulfamethoxazole against community-associated methicillin-resistant Staphylococcus aureus. J Antimicrob Chemother. 2008;62(6):1305-10 (Epub 2008/09/20).

20. Werth BJ, Sakoulas G, Rose WE, Pogliano J, Tewhey $\mathrm{R}$, Rybak MJ. Ceftaroline increases membrane binding and enhances the activity of daptomycin against daptomycin-nonsusceptible vancomycinintermediate Staphylococcus aureus in a pharmacokinetic/pharmacodynamic model. Antimicrob Agents Chemother. 2013;57(1):66-73 (Epub 2012/10/17). 